\title{
Stability of soliton families in nonlinear Schrödinger equations with non-parity-time-symmetric complex potentials
}

\author{
Jianke Yang*, Sean Nixon \\ Department of Mathematics and Statistics, \\ University of Vermont, Burlington, VT 05401 \\ Corresponding author email: jyang@math.uvm.edu
}

\begin{abstract}
Stability of soliton families in one-dimensional nonlinear Schrödinger equations with non-parity-time $(\mathcal{P T})$-symmetric complex potentials is investigated numerically. It is shown that these solitons can be linearly stable in a wide range of parameter values both below and above phase transition. In addition, a pseudo-Hamiltonian-Hopf bifurcation is revealed, where pairs of purely-imaginary eigenvalues in the linear-stability spectra of solitons collide and bifurcate off the imaginary axis, creating oscillatory instability, which resembles Hamiltonian-Hopf bifurcations of solitons in Hamiltonian systems even though the present system is dissipative and non-Hamiltonian. The most important numerical finding is that, eigenvalues of linear-stability operators of these solitons appear in quartets $\left(\lambda,-\lambda, \lambda^{*},-\lambda^{*}\right)$, similar to conservative systems and $\mathcal{P} \mathcal{T}$-symmetric systems. This quartet eigenvalue symmetry is very surprising for non- $\mathcal{P} \mathcal{T}$-symmetric systems, and it has far-reaching consequences on the stability behaviors of solitons.
\end{abstract}

Keywords: non- $\mathcal{P} \mathcal{T}$-symmetric potentials, soliton families, stability, NLS equations

\section{Introduction}

Parity-time $(\mathcal{P T})$ symmetry is currently at the forefront of research in physics and applied mathematics (see $[1,2]$ for reviews). This concept started out in quantum mechanics, where it was observed that complex potentials with parity-time symmetry could still exhibit all-real spectra even though the underlying Schrödinger operator is non-Hermitian [3]. Later, this concept spread to optics, where it was realized that optical waveguides with even refractive-index profiles and odd gainloss distributions constitute $\mathcal{P} \mathcal{T}$-symmetric systems [4]. In this optical setting, $\mathcal{P} \mathcal{T}$ symmetry was observed for the first time [5-7]. In addition, it has been introduced into many other physical disciplines such as Bose-Einstein condensates, electronic circuits and mechanical systems [8-13]. $\mathcal{P} \mathcal{T}$ systems feature a unique property - phase transition, where the linear spectrum changes from all-real to partially-complex when the system parameters cross a certain threshold [3, 4, 14, 15]. This phase transition has led to interesting applications such as single-mode $\mathcal{P} \mathcal{T}$ lasers and unidirectional reflectionless optical devices [16-18]. A surprising property of $\mathcal{P} \mathcal{T}$ systems is that, even though they are dissipative due to the gain and loss, they exhibit many properties of conservative systems, such as all-real linear spectra and continuous families of stationary nonlinear modes $[3,4,9,14,15,19-26]$. Thus, $\mathcal{P} \mathcal{T}$ systems break the boundary between conservative and dissipative systems and offer novel wave-guiding possibilities. In addition, $\mathcal{P} \mathcal{T}$ systems make loss useful, which is enlightening.

The downside of $\mathcal{P} \mathcal{T}$ symmetry stems from the restrictive conditions set on the gain-loss profile, which must be odd. To overcome this restriction, non- $\mathcal{P} \mathcal{T}$-symmetric dissipative systems sharing the properties of $\mathcal{P} \mathcal{T}$-symmetric systems have been pursued. For instance, wide classes of non- $\mathcal{P} \mathcal{T}$-symmetric potentials with all-real spectra were reported in [27-29, 31, 32]. In addition, it was discovered that in a certain class of such potentials with the form $g^{2}(x)+i g^{\prime}(x)$, where $g(x)$ is an arbitrary real function, solitons also appear as continuous families, which is very counter-intuitive [31, 33-35]. Furthermore, it was argued in [35] that potentials of the form $g^{2}(x)+i g^{\prime}(x)$ are the only one-dimensional (1D) non- $\mathcal{P} \mathcal{T}$-symmetric potentials which support soliton families. However, stability properties of these soliton families are still largely unknown, except for some evolution simulations of perturbed simple-shaped solitons in a certain non- $\mathcal{P} \mathcal{T}$-symmetric potential below a phase transition in [31], which suggest that those simple solitons could be stable.

In this paper, we systematically study the linear stability of various soliton families in 1D nonlinear Schrödinger (NLS) equations with non- $\mathcal{P} \mathcal{T}$-symmetric complex potentials both below and above phase transition. This study is performed by numerically computing the linear-stability spectra of these solitons. We show that both simple-shaped and multi-humped soliton families can be linearly stable in a wide range of parameter values below and above a phase transition. In addition, a 
pseudo-Hamiltonian-Hopf bifurcation is revealed, where pairs of purely-imaginary eigenvalues in the linear-stability spectra of solitons collide and bifurcate off the imaginary axis, creating oscillatory instability, which resembles Hamiltonian-Hopf bifurcations of solitons in Hamiltonian systems even though the present system is non-Hamiltonian. Our most important numerical finding is that, eigenvalues of the linear-stability operator of these solitons appear in quartets $\left(\lambda,-\lambda, \lambda^{*},-\lambda^{*}\right)$, similar to conservative systems and $\mathcal{P} \mathcal{T}$-symmetric systems. This quartet eigenvalue symmetry is very surprising for non- $\mathcal{P} \mathcal{T}$-symmetric dissipative systems, and its consequences on the linear stability of these solitons are explained.

\section{Preliminaries}

The mathematical model we consider is the following NLS equation with an external potential

$$
i \Psi_{t}+\Psi_{x x}+V(x) \Psi+\sigma|\Psi|^{2} \Psi=0
$$

where $V(x)$ is a complex potential, and $\sigma= \pm 1$ is the sign of nonlinearity. This model governs nonlinear light propagation in an optical medium with gain and loss [4, 36, 37], as well as dynamics of Bose-Einstein condensates in a double-well potential with atoms injected into one well and removed from the other well $[11,12,38]$. If the potential $V(x)$ is real, Eq. (2.1) is conservative and Hamiltonian, and its properties have been investigated in numerous articles for many decades [36, 37]. If $V(x)$ is complex but $\mathcal{P} \mathcal{T}$-symmetric, i.e., $V^{*}(x)=V(-x)$, where the superscript $*$ represents complex conjugation, then this $\mathcal{P} \mathcal{T}$-symmetric system has been heavily studied in the last eight years [1, 2]. If $V(x)$ is complex and non- $\mathcal{P} \mathcal{T}$-symmetric, this equation is currently at the frontier of research. For non$\mathcal{P T}$-symmetric potentials of the form

$$
V(x)=g^{2}(x)+2 \gamma g(x)+i g^{\prime}(x),
$$

where $g(x)$ is a real asymmetric function and $\gamma$ a real constant, the linear spectrum of the potential can be all-real, which is unusual [30-32]. Note that this form of the potential is equivalent to $g^{2}(x)+i g^{\prime}(x)$ under a shift $g(x)+\gamma \rightarrow g(x)$ and a gauge transformation to Eq. (2.1). It is used in this article since it is more convenient to induce a phase transition by varying the parameter $\gamma$ while keeping the function $g(x)$ fixed. A more important phenomenon with the potential (2.2) is that, Eq. (2.1) under this potential admits continuous families of solitons [31, 34, 35]. This is surprising since, in typical dissipative systems, solitons exist as isolated solutions with discrete power levels due to the requirement of balance between gain and loss [39]. Dissipative but $\mathcal{P} \mathcal{T}$-symmetric systems admit soliton families with continuous power levels, which is interesting [9, 14, 19-24]. However, the existence of such soliton families can be easily understood due to the $\mathcal{P} \mathcal{T}$ symmetry, which assures the balancing of gain and loss for all $\mathcal{P} \mathcal{T}$-symmetric solitons [40]. Soliton families in non- $\mathcal{P} \mathcal{T}$-symmetric systems, on the other hand, are much less obvious, and their existence has yet to be fully understood.

Solitons in Eq. (2.1) are of the form

$$
\Psi(x, t)=e^{-i \mu t} \psi(x)
$$

where $\mu$ is a real propagation constant, and $\psi(x)$ is a localized function satisfying the equation

$$
\psi_{x x}+\mu \psi+V(x) \psi+\sigma|\psi|^{2} \psi=0
$$

For the complex non- $\mathcal{P} \mathcal{T}$-symmetric potential (2.2), these solitons exist as continuous families, and they can be computed by various numerical methods such as the squared-operator method and the Newton-conjugate-gradient method [36]. To study their linear stability, we perturb these solitons by infinitesimal normal modes,

$$
\Psi(x, t)=e^{-i \mu t}\left[\psi(x)+f_{1}(x) e^{\lambda t}+f_{2}^{*}(x) e^{\lambda^{*} t}\right],
$$

where $\left|f_{1}\right|,\left|f_{2}\right| \ll|\psi|$. Substituting this perturbation into Eq. (2.1) and linearizing, we obtain a linear-stability eigenvalue problem

$$
L\left(\begin{array}{l}
f_{1} \\
f_{2}
\end{array}\right)=\lambda\left(\begin{array}{l}
f_{1} \\
f_{2}
\end{array}\right)
$$

where

$$
L=\left(\begin{array}{cc}
L_{11} & L_{12} \\
L_{12}^{*} & L_{11}^{*}
\end{array}\right)
$$

and

$$
L_{11}=i\left[\partial_{x x}+\mu+V(x)+2 \sigma|\psi|^{2}\right], \quad L_{12}=i \sigma \psi^{2}
$$

This eigenvalue problem can be computed by the Fourier collocation method (for the full spectrum) or the Newton-conjugategradient method (for individual discrete eigenvalues) [36]. If eigenvalues with positive real parts exist, the soliton is linearly (spectrally) unstable; otherwise it is linearly (spectrally) stable.

Symmetry properties of the linear-stability operator $L$ and its eigenvalues are important since they strongly influence the stability results. If the potential $V(x)$ is real [i.e., when Eq. (2.1) is Hamiltonian], then $L$ satisfies the following two symmetry relations,

$$
\begin{gathered}
L^{*}=\sigma_{1} L \sigma_{1}^{-1}, \\
L^{\dagger}=-\sigma_{3} L \sigma_{3}^{-1},
\end{gathered}
$$

where the superscript $\dagger$ represents the Hermitian (conjugate transpose) of a matrix operator, and

$$
\sigma_{1}=\left[\begin{array}{ll}
0 & 1 \\
1 & 0
\end{array}\right], \quad \sigma_{3}=\left[\begin{array}{cc}
1 & 0 \\
0 & -1
\end{array}\right]
$$

are the first and third Pauli spin matrices. The similarity relation (2.8) shows that $L^{*}$ and $L$ share the same spectrum. Then, since the spectrum of $L^{*}$ is also the complex conjugate of the spectrum of $L$, we see that eigenvalues of $L$ must come in conjugate 
pairs $\left(\lambda, \lambda^{*}\right)$. The symmetry relation (2.9) shows that the spectrum of $L^{\dagger}$ is negative of the spectrum of $L$. Since the spectrum of $L^{\dagger}$ is also complex conjugate of the spectrum of $L$, eigenvalues of $L$ then must come in pairs of $\left(\lambda,-\lambda^{*}\right)$. Combining these two eigenvalue symmetries, we conclude that for real potentials (Hamiltonian systems), complex eigenvalues of $L$ must come in quartets $\left(\lambda,-\lambda, \lambda^{*},-\lambda^{*}\right)$, which is a well-known fact. In the special case when the eigenvalue $\lambda$ is real or purely-imaginary, this quartet symmetry reduces to a pair symmetry $(\lambda,-\lambda)$.

It is noted that in a real potential $V(x)$, if the soliton $\psi(x)$ is also real (which is generally the case), then $L^{*}=-L$. Using this symmetry, instead of (2.9), one can also show that eigenvalues of $L$ come in pairs of $\left(\lambda,-\lambda^{*}\right)$. However, for real potentials $V$ in higher spatial dimensions, if the soliton $\psi$ is complex (such as a vortex soliton), then the symmetry $L^{*}=-L$ would not hold, but (2.9) still does.

If the potential $V(x)$ is complex but $\mathcal{P} \mathcal{T}$-symmetric, then the symmetry relation $(2.8)$ persists, but the other relation (2.9) breaks down. In this case, if the soliton $\psi(x)$ is also $\mathcal{P} \mathcal{T}$ symmetric, i.e., $\psi^{*}(x)=\psi(-x)$, then another symmetry relation

$$
L^{*}=-\mathcal{P} L \mathcal{P}^{-1}
$$

is valid, where $\mathcal{P}$ is the parity operator, i.e., $\mathcal{P} f(x) \equiv f(-x)$. Utilizing the two symmetry relations (2.8) and (2.10) and repeating the above arguments, we conclude that for $\mathcal{P} \mathcal{T}$-symmetric solitons in $\mathcal{P} \mathcal{T}$-symmetric potentials, complex eigenvalues of $L$ must also come in quartets $\left(\lambda,-\lambda, \lambda^{*},-\lambda^{*}\right)$. This fact has been pointed out in [41] although it may not be widely known.

If the potential $V(x)$ is complex and non- $\mathcal{P} \mathcal{T}$-symmetric, the symmetry relation (2.8) still holds, but we cannot see any additional symmetry for $L$. This suggests that in this case, complex eigenvalues of $L$ may only appear in conjugate pairs $\left(\lambda, \lambda^{*}\right)$, but not in quartets. However, a remarkable discovery from our numerics in the later text is that for non- $\mathcal{P} \mathcal{T}$-symmetric potentials of the form (2.2), eigenvalues of $L$ still appear as quartets $\left(\lambda,-\lambda, \lambda^{*},-\lambda^{*}\right)$, just as in Hamiltonian and $\mathcal{P} \mathcal{T}$-symmetric systems. This quartet eigenvalue symmetry has important consequences on the stability of solitons, which will be described later in this article.

Our stability analysis will be performed by numerically computing the spectrum of the linear-stability operator $L$. This spectrum will be obtained by the Fourier-collocation method [36]. Discrete eigenvalues in this spectrum are further checked by the Newton-conjugate-gradient method [36]. Both methods can yield eigenvalues with accuracy of $10^{-10}$ or higher. These linear-stability results will also be corroborated by direct evolution simulations of these solitons under initial random-noise perturbations using the pseudo-spectral method [36]. In our numerical examples, we take

$$
g(x)=\tanh 2(x+2.5)-\tanh (x-2.5),
$$

which is an asymmetric single-hump function. For this choice of $g(x)$, a phase transition occurs at $\gamma=\gamma_{c} \approx-0.1806$, where the linear spectrum of the potential is all-real when $\gamma>\gamma_{c}$ and becomes partially complex when $\gamma<\gamma_{c}$.

\section{Linear stability of solitons below phase transition}

First, we consider the linear stability of soliton families below phase transition. For this purpose, we take $\gamma=0$. The resulting potential $V(x)$ and its linear spectrum are displayed in Fig. 1. Notice that the real part of $V(x)$ is not even, and its imaginary part not odd, thus this potential is non- $\mathcal{P} \mathcal{T}$-symmetric, but its spectrum is all-real. In addition, this spectrum contains three discrete eigenvalues,

$$
\mu_{1} \approx-3.4484, \quad \mu_{2} \approx-2.1899, \quad \mu_{3} \approx-0.7044
$$
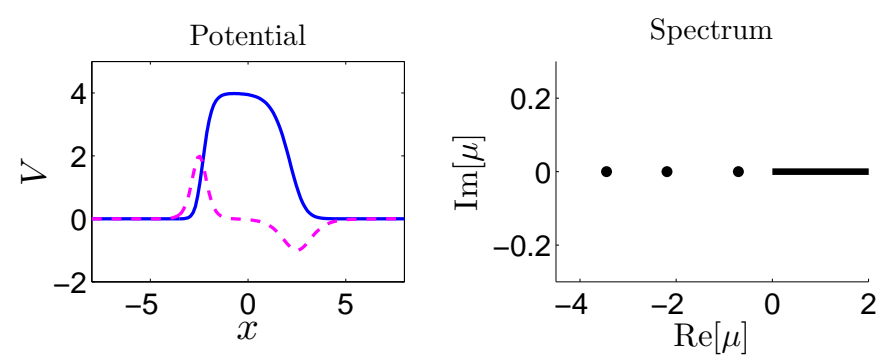

Figure 1: (Color online) The complex potential (2.2) with $g(x)$ given in (2.11) and $\gamma=0$ (left) and its linear spectrum (right).

Continuous families of solitons can bifurcate out from each of these three discrete eigenvalues under either sign of nonlinearity. We first consider the soliton family bifurcating from the first (lowest) eigenvalue $\mu=\mu_{1}$ under focusing nonlinearity $(\sigma=1)$. Defining the power of a soliton as $P=\int_{-\infty}^{\infty}|\psi|^{2} d x$, the power curve of this soliton family is plotted in Fig. 2(a). At the marked point of this power curve (with $\mu=-5$ ), the amplitude profile of the soliton is displayed in Fig. 2(b). It is seen that this amplitude profile is single-humped. Since this soliton family bifurcates from the lowest eigenvalue $\mu_{1}$, we call this family of solitons fundamental solitons. We have computed the linear-stability spectra for these fundamental solitons, and found that their eigenvalues all lie on the imaginary axis. Thus, these fundamental solitons are linearly stable. As an example, the linear-stability spectrum for the soliton of Fig. 2(b) is shown in Fig. 2(c). This spectrum consists of three pairs of discrete non-zero eigenvalues and the continuous spectrum, all on the imaginary axis. Time evolution of this soliton for 200 time units under initial $1 \%$ random noise perturbations is plotted in Fig. 2(d). It is seen that this soliton is robust against perturbations, consistent with its linear-stability result.

Next, we consider the soliton family bifurcating from the second eigenvalue $\mu=\mu_{2}$ under focusing nonlinearity. The power curve of this soliton family is displayed in Fig. 3(a). At the marked points ' $c$, $d$ ' of this power curve (with $\mu=-2.34$ 

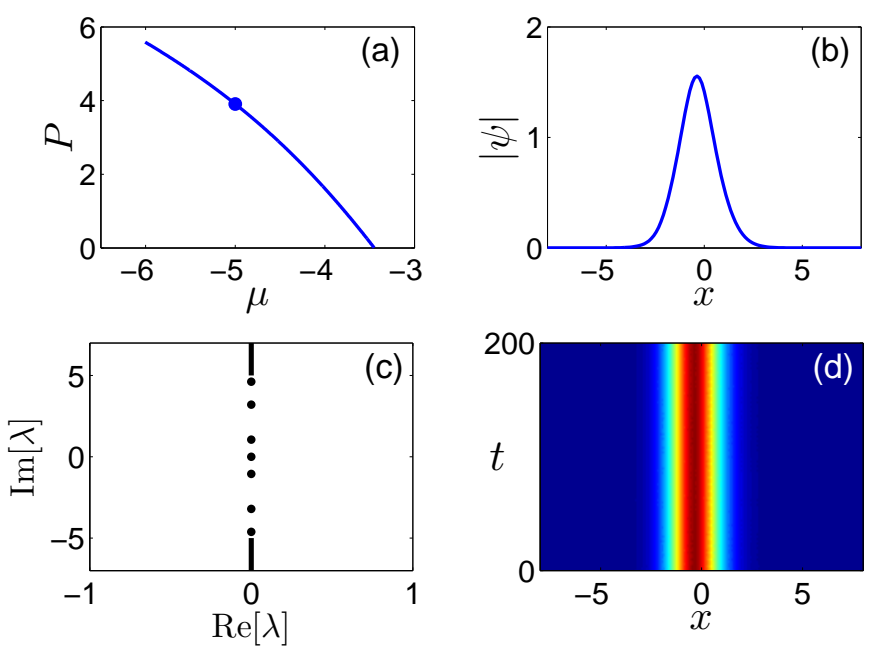

Figure 2: (Color online) (a) Power curve of fundamental solitons (bifurcating from the eigenvalue $\mu_{1}$ ) below phase transition under focusing nonlinearity. (b) Amplitude profile of the soliton at the marked point of the power curve. (c) Linear-stability spectrum for the soliton in (b). (d) Time evolution of the soliton in (b) under $1 \%$ random-noise perturbations.

and -2.55 respectively), amplitude profiles of the solitons are shown in Fig. 3(b). These profiles are double-humped, indicating that this family of solitons are excited states. At low powers, these solitons are linearly stable. This is evidenced by the linear-stability spectrum shown in Fig. 3(c) for the lower-power soliton in Fig. 3(b), where all eigenvalues are purely imaginary. However, at higher powers, these excited-state solitons become linearly unstable. This can be seen from the linear-stability spectrum in Fig. 3(d) for the higher-power soliton in Fig. 3(b). In this spectrum, a quartet of complex eigenvalues appear, creating oscillatory instability. To corroborate these linear-stability results, we have simulated the evolutions of the two solitons in Fig. 3(b) under 1\% random-noise perturbations, and the simulation results are displayed in Fig. 3(e,f). The panel (e) shows robust (stable) propagation, while the panel (f) shows the onset of oscillatory instability, consistent with the linear-stability results.

The change of linear stability in this family of excited-state solitons occurs at $\mu=\mu_{c} \approx-2.440$, where $P_{c} \approx 0.925$. This instability arises when two pairs of imaginary eigenvalues bifurcate into a complex quartet. Below the critical power $P_{c}$, the stability spectrum of the soliton contains two pairs of imaginary eigenvalues $\left( \pm i \omega_{1}, \pm i \omega_{2}\right)$ [see Fig. 3(c)]. As the soliton's power increases to $P_{c}$, eigenvalues $\pm i \omega_{1}$ and $\pm i \omega_{2}$ approach each other on the imaginary axis. At the critical power $P_{c}$, these imaginary eigenvalues coalesce and form exceptional points with geometric multiplicity one and algebraic multiplicity two. Above the critical power $P_{c}$, these exceptional points bifurcate off the imaginary axis, creating a quartet of complex eigenvalues (and
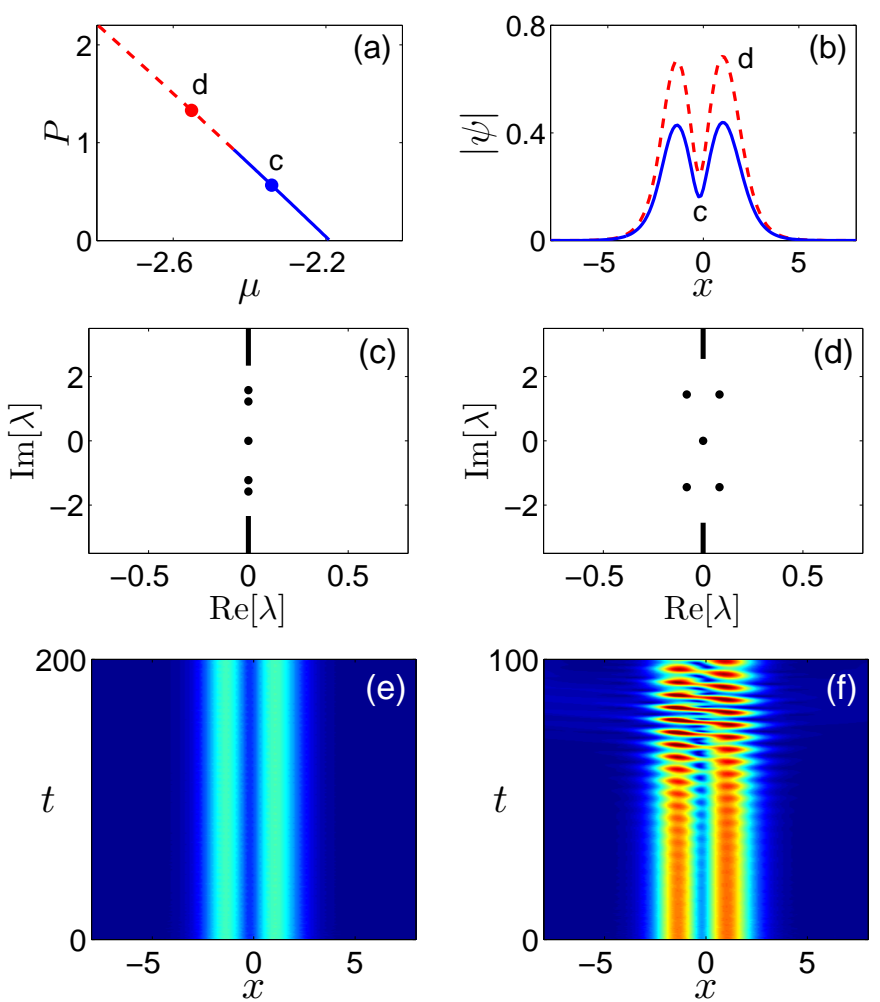

Figure 3: (Color online) (a) Power curve of excited-state solitons (bifurcating from the eigenvalue $\mu_{2}$ ) below phase transition under focusing nonlinearity (solid blue indicates stability, and dashed red indicates instability). (b) Amplitude profiles of solitons at the marked points ' $c$, d' of the power curve (lower for 'c' and upper for 'd'). (c, d) Linear-stability spectra for the lower and upper solitons in panel (b) respectively. (e, f) Time evolutions of the lower and upper solitons in panel (b) under $1 \%$ random-noise perturbations respectively.

hence oscillatory instability) [see Fig. 3(d)]. This instability mechanism is remarkably similar to Hamiltonian-Hopf bifurcations in Hamiltonian systems [such as when the potential $V(x)$ in Eq. (2.1) is real] [42, 43], even though the present system is non-Hamiltonian. Thus, we call this change of linear stability pseudo-Hamiltonian-Hopf bifurcation.

We note that pseudo-Hamiltonian-Hopf bifurcations can appear in $\mathcal{P} \mathcal{T}$-symmetric systems as well. This fact has not been reported in the literature yet, but we have seen it in our numerics of $\mathcal{P} \mathcal{T}$-symmetric systems [such as in Eq. (2.1) with a $\mathcal{P T}$-symmetric potential].

A remarkable feature in the above stability results is that, although Eq. (2.1) in our consideration is non-Hamiltonian and non- $\mathcal{P} \mathcal{T}$-symmetric, and the gain and loss in the system are rather large, stability behaviors of solitons in our system are analogous to those in Hamiltonian systems. Examples include the stability of fundamental solitons and pseudo-HamiltonianHopf bifurcations of excited-state solitons [42-44]. However, differences in behaviors between our system and Hamiltonian 
systems also exist. For instance, in our system, low-amplitude fundamental solitons can be linearly unstable, and pseudoHamiltonian-Hopf bifurcations can occur on fundamental solitons at higher powers. These phenomena would not happen in Hamiltonian systems. These differences will be shown in the next section.

\section{Linear stability of solitons above phase transition}

Now we consider the stability of solitons above phase transition. For this purpose, we take $\gamma=-0.3$. The resulting potential (2.2) is displayed in Fig. 4. Clearly, this potential is also non- $\mathcal{P} \mathcal{T}$-symmetric. When compared to the potential in Fig. 1, the real part of this potential is significantly lower, while its imaginary part remains the same. The linear spectrum of this potential, displayed also in Fig. 4, shows the presence of a pair of complex eigenvalues, indicating that this potential is above phase transition. It is noted that for complex potentials of the form (2.2), complex eigenvalues in the linear spectrum of the potential appear as conjugate pairs $\left(\mu, \mu^{*}\right)$ [32]. This eigenvalue symmetry is clearly visible in Fig. 4.
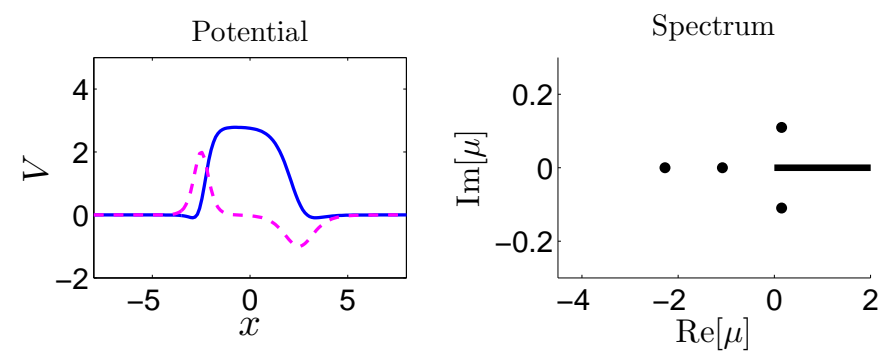

Figure 4: (Color online) The complex potential (2.2) with $g(x)$ given in (2.11) and $\gamma=-0.3$ (left) and its linear spectrum (right).

In addition to the pair of complex eigenvalues, the spectrum in Fig. 4 also contains two discrete real eigenvalues,

$$
\mu_{1} \approx-2.2740, \quad \mu_{2} \approx-1.0787
$$

From these real eigenvalues, soliton families can bifurcate out under either sign of nonlinearity. Here, we consider the soliton family bifurcating from the lowest eigenvalue $\mu_{1}$ under focusing nonlinearity $(\sigma=1)$. The power curve of this soliton family is plotted in Fig. 5(a), and the solitons at the marked points ' $c$, d' of the power curve, with $\mu=-2.5$ and -3.3 respectively, are shown in Fig. 5(b). These solitons have a single-hump amplitude profile, and are fundamental solitons due to their bifurcation from the lowest eigenvalue $\mu_{1}$.

At low powers, these solitons are linearly unstable because the underlying potential is above phase transition. This is evidenced in Fig. 5(c), where the linear-stability spectrum for the lower-power soliton in Fig. 5(b) is displayed. This instability is due to a quartet of complex eigenvalues $\left(\lambda_{0}, \lambda_{0}^{*},-\lambda_{0},-\lambda_{0}^{*}\right)$, which are directly related to the pair of complex eigenvalues in the linear spectrum of the potential in Fig. 4. However, at higher powers, these solitons become linearly stable. This is evidenced in Fig. 5(d), where the linear-stability spectrum for the higherpower soliton of Fig. 5(b) is plotted. All eigenvalues in this spectrum are on the imaginary axis, indicating this soliton is linearly stable. The change of stability occurs at $\mu=\mu_{c} \approx-2.86$, where $P_{c} \approx 1.74$.
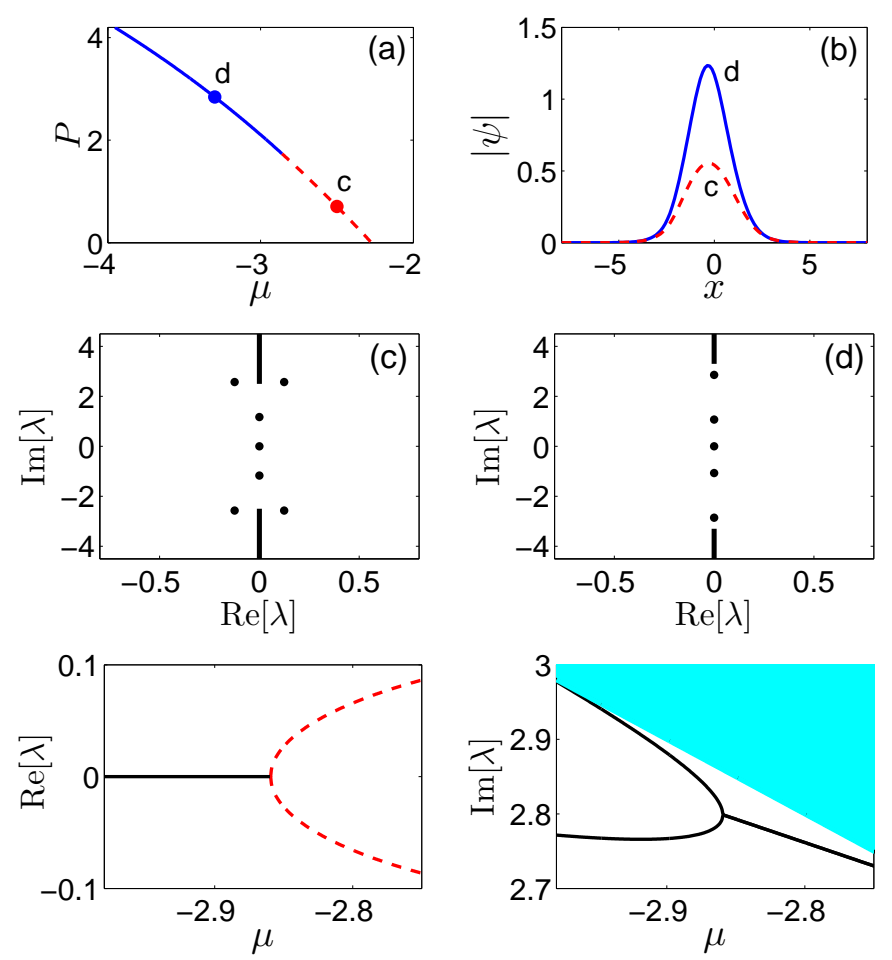

Figure 5: (Color online) (a) Power curve of fundamental solitons (bifurcating from the eigenvalue $\mu_{1}$ ) above phase transition under focusing nonlinearity (solid blue indicates stability, and dashed red indicates instability). (b) Amplitude profiles of solitons at the marked points ' $c$, d' of the power curve (lower for 'c' and upper for 'd'). (c, d) Linear-stability spectra for the lower and upper solitons in panel (b) respectively. (e, f) Real and imaginary parts of linear-stability eigenvalues $\lambda$ versus the propagation constant $\mu$ (the continuous spectrum is shown in light blue).

The reason for this stabilization of solitons at higher powers is that, as the power increases toward the critical power $P_{c}$, the quartet of complex eigenvalues $\left(\lambda_{0}, \lambda_{0}^{*},-\lambda_{0},-\lambda_{0}^{*}\right)$ move toward the imaginary axis. At the critical power $P_{c}$, these complex eigenvalues collide on the imaginary axis and create a pair of exceptional points. Above the critical power, these exceptional points split along the imaginary axis and become two pairs of imaginary eigenvalues $\left\{ \pm i \omega_{1}, \pm i \omega_{2}\right\}$, thus the solitons become linearly stable. This stabilization process is more clearly depicted in Fig. 5(e,f), where the real and imaginary parts of the relevant linear-stability eigenvalues are plotted versus the propagation constant $\mu$. This stabilization is a reverse pseudo- 
Hamiltonian-Hopf bifurcation as the power rises.

In this example, fundamental solitons at low powers are linearly unstable, and a reverse pseudo-Hamiltonian-Hopf bifurcation is seen. These phenomena will not occur in Hamiltonian systems, such as Eq. (2.1) with a real potential. In such Hamiltonian systems, fundamental solitons at low amplitudes are always linearly and nonlinearly stable because their HamiltonianKrein index is zero [44]. In addition, Hamiltonian-Hopf bifurcations cannot occur on fundamental solitons of any powers, because there are no imaginary eigenvalues with negative Krein signatures, but such imaginary eigenvalues are necessary for Hamiltonian-Hopf bifurcations [44].

\section{Quartet eigenvalue symmetry and its consequences}

The most surprising finding of the above stability analysis is that, linear-stability eigenvalues of solitons in non- $\mathcal{P} \mathcal{T}$ symmetric potentials (2.2) appear in quartets $\left(\lambda, \lambda^{*},-\lambda,-\lambda^{*}\right)$, i.e., if $\lambda$ is an eigenvalue of the operator $L$, so are $\lambda^{*},-\lambda$ and $-\lambda^{*}$. As we have pointed out in Sec. 2 , in non- $\mathcal{P} \mathcal{T}$-symmetric potentials (2.2), linear-stability eigenvalues still appear in pairs $\left(\lambda, \lambda^{*}\right)$, i.e., the spectrum is symmetric with respect to the real axis. But we cannot see another symmetry of the operator $L$ which assures the appearance of eigenvalues in $\left(\lambda,-\lambda^{*}\right)$ pairs, i.e., the spectrum's symmetry with respect to the imaginary axis. Because of this, we do not anticipate the quartet eigenvalue symmetry in the spectrum of $L$. However, our numerical results in the earlier text show that these eigenvalues do come in quartets of $\left(\lambda, \lambda^{*},-\lambda,-\lambda^{*}\right)$, which is very remarkable.

The visual evidence of this quartet eigenvalue symmetry can already be seen in the stability spectra of Figs. 2, 3 and 5, where the spectra are always symmetric with respect to both the real and imaginary axes. Here, we establish this eigenvalue symmetry quantitatively. Since the $\left(\lambda, \lambda^{*}\right)$ symmetry is already known, we focus on the $\left(\lambda,-\lambda^{*}\right)$ symmetry below. To establish this latter symmetry, we first consider the two upper complex eigenvalues in Fig. 3(d). Numerical computations give these two eigenvalues (accurate to all twelve digits) as

$$
\begin{aligned}
& \lambda_{1}=-0.08220738069 \ldots+1.43969109965 \ldots i, \\
& \lambda_{2}=0.08220738069 \ldots+1.43969109965 \ldots i .
\end{aligned}
$$

Clearly, $\lambda_{2}=-\lambda_{1}^{*}$ to numerical accuracy. Using multi-precision computation, we have further checked that $\lambda_{2}$ and $-\lambda_{1}^{*}$ match each other to many more digits. The eigenfunctions $\left(f_{1}, f_{2}\right)$ of these two eigenvalues are plotted in Fig. 6. Notice that these two eigenfunctions are not related to each other by any obvious symmetry. However, their eigenvalues are related as $\lambda_{2}=-\lambda_{1}^{*}$, which is quite intriguing.

As another example, we consider the two upper complex eigenvalues in the spectrum of Fig. 5(c). Numerical computations give these two eigenvalues (accurate to all twelve digits) as

$$
\lambda_{1}=-0.12447936624 \ldots+2.57218047717 \ldots i,
$$
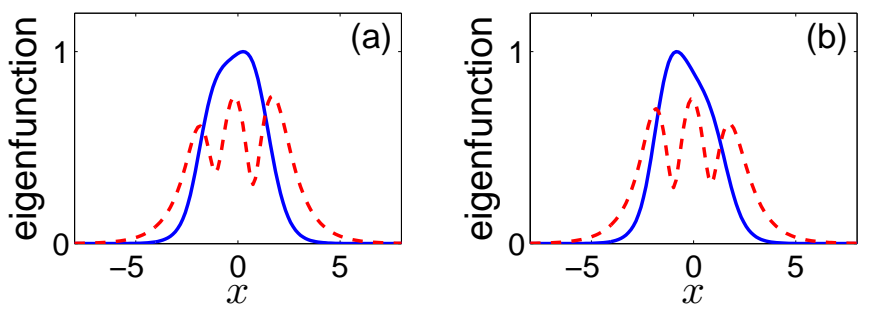

Figure 6: (Color online) Eigenfunctions $\left(f_{1}, f_{2}\right)$ for the upper two complex eigenvalues in the spectrum of Fig. 3(d), with $\lambda=\lambda_{1}$ in (a) and $\lambda=\lambda_{2}$ in (b), where $\lambda_{1,2}$ are given in (5.1)-(5.2). Solid blue lines are $\left|f_{1}\right|$ and dashed red lines $\left|f_{2}\right|$.

$$
\lambda_{2}=0.12447936624 \ldots+2.57218047717 \ldots i .
$$

Again, $\lambda_{2}=-\lambda_{1}^{*}$ to numerical accuracy.

We have examined the other eigenvalues in the spectra of Figs. 2, 3 and 5, and found them to lie exactly on the imaginary axis (to high numerical accuracy). Thus, these spectra are indeed symmetric with respect to both the real and imaginary axes, confirming the quartet eigenvalue symmetry of $\left(\lambda, \lambda^{*},-\lambda,-\lambda^{*}\right)$

In addition to the stability spectra in Figs. 2, 3 and 5, we have examined the spectra of solitons in other non- $\mathcal{P} \mathcal{T}$-symmetric potentials of the form (2.2), which are not included in this article. Those stability spectra respect the quartet eigenvalue symmetry as well.

This quartet eigenvalue symmetry in the linear-stability spectrum has far reaching consequences on the linear-stability behaviors of solitons. First, it assures the linear stability of lowpower fundamental solitons (bifurcating from the lowest discrete eigenvalue of the potential) as long as the potential is below phase transition. This follows from the fact that a potential which is below phase transition has all-real spectra and its discrete non-zero eigenvalues can be assumed to be all simple (which is the generic case). Then, in the limit of zero amplitude of fundamental solitons, the linear-stability spectrum (of operator $L$ ) is purely imaginary, and all discrete non-zero eigenvalues of $L$ are simple. In addition, no discrete eigenvalues are embedded inside the continuous spectrum. When the amplitude of the soliton is non-zero but small, by virtue of the eigenvalue continuity and quartet eigenvalue symmetry, the simple discrete imaginary eigenvalues of $L$ cannot move off the imaginary axis. Meanwhile, the zero eigenvalue and the continuous spectrum do not change. Thus, the spectrum remains on the imaginary axis, and low-amplitude fundamental solitons are linearly stable below phase transition. This analytically explains our numerical findings for low-power solitons in Fig. 2.

By a similar argument, we can also show that, in the presence of this quartet eigenvalue symmetry, low-power excited-state solitons (bifurcating from the higher discrete real eigenvalues $\mu_{k}$ of the potential with $k>1$ ) are also linearly stable if the potential is below phase transition, and none of $i\left(\mu_{k}-\mu_{j}\right)(j \neq k)$ 
is embedded inside the continuous spectrum of operator $L$ when $\psi(x)=0$. For the example in Fig. 3, the latter condition means $\left|\mu_{2}-\mu_{j}\right|<\left|\mu_{2}\right|(j=1,3)$, which is satisfied. This analytically explains the linear stability of low-power excited-state solitons below phase transition in Fig. 3.

Another consequence of this quartet eigenvalue symmetry is that it makes pseudo-Hamiltonian-Hopf bifurcation possible in the non-Hamiltonian system (2.1). If the linear-stability spectrum contains two pairs of simple imaginary eigenvalues $\left\{ \pm i \omega_{1}, i \omega_{2}\right\}$ for a certain soliton, then when the propagation constant of the soliton continuously changes, these simple imaginary eigenvalues have to stay on the imaginary axis due to the quartet eigenvalue symmetry. In this case, if these eigenvalues move toward each other and collide $\left(\omega_{1} \rightarrow \omega_{2}\right)$, they could leave the imaginary axis and become a quartet of complex eigenvalues, creating a pseudo-Hamiltonian-Hopf bifurcation. This is exactly what happens in Figs. 3 and Fig. 5.

One more consequence of this quartet eigenvalue symmetry is that it closely mimics that of solitons in Hamiltonian systems and of $\mathcal{P} \mathcal{T}$-symmetric solitons in $\mathcal{P} \mathcal{T}$-symmetric systems (see Sec. 2). This implies that non- $\mathcal{P} \mathcal{T}$-symmetric solitons in complex potentials (2.2) are likely to share many stability properties of those other systems, as the results of this paper have shown.

\section{Summary and discussion}

In this paper, we have numerically analyzed the linear stability of soliton families in 1D NLS equations (2.1) with non$\mathcal{P} \mathcal{T}$-symmetric complex potentials (2.2). We have shown that these solitons can be linearly stable in a wide range of parameter values both below and above phase transition. More importantly, we have discovered that linear-stability eigenvalues of these solitons appear in quartets $\left(\lambda,-\lambda, \lambda^{*},-\lambda^{*}\right)$, similar to conservative systems and $\mathcal{P} \mathcal{T}$-symmetric systems. This quartet eigenvalue symmetry is very surprising for non- $\mathcal{P} \mathcal{T}$-symmetric systems, and it facilitates the existence of stable solitons and makes their pseudo-Hamiltonian-Hopf bifurcation possible.

A question closely related to the subject of this paper is the linear stability of asymmetric solitons in $\mathcal{P} \mathcal{T}$-symmetric potentials. Earlier work has shown that in $\mathcal{P} \mathcal{T}$-symmetric potentials of the same form (2.2) [where $g(x)$ is now an even function], symmetry breaking of solitons can occur [45]. That is, from the base branch of $\mathcal{P} \mathcal{T}$-symmetric solitons, a branch of asymmetric solitons can bifurcate out. The linearization operator $L$ of these asymmetric solitons only admits the symmetry (2.8), as far as one can see, similar to the present case. This $L$ symmetry only assures its eigenvalue symmetry of $\left(\lambda, \lambda^{*}\right)$. However, our numerical studies (not shown in this article) have revealed that, their linear-stability eigenvalues also appear in quartets of $\left(\lambda,-\lambda, \lambda^{*},-\lambda^{*}\right)$, closely resembling the findings in this article for non- $\mathcal{P} \mathcal{T}$-symmetric potentials (2.2). This quartet eigenvalue symmetry for asymmetric solitons in $\mathcal{P} \mathcal{T}$-symmetric potentials (2.2) is equally surprising.
Another question closely related to the subject of this paper is the linear stability of two-dimensional solitons in non$\mathcal{P} \mathcal{T}$-symmetric complex potentials. Earlier work has shown that in two-dimensional non- $\mathcal{P} \mathcal{T}$-symmetric complex potentials of certain forms, continuous families of solitons can also bifurcate out from linear modes [41]. Our numerical studies (not included in this article) have found that linearstability eigenvalues of those $2 \mathrm{D}$ solitons only possess the conjugate-pair symmetry of $\left(\lambda, \lambda^{*}\right)$, but NOT the quartet symmetry of $\left(\lambda,-\lambda, \lambda^{*},-\lambda^{*}\right)$. This result echoes that for linearstability eigenvalues of asymmetric solitons in 2D partially$\mathcal{P T}$-symmetric potentials [41]. These results indicate that the quartet eigenvalue symmetry depends on the spatial dimension of the problem.

Many questions are still open regarding the findings of this paper. The most important question is why the quartet eigenvalue symmetry appears for linear-stability eigenvalues of solitons in non- $\mathcal{P} \mathcal{T}$-symmetric complex potentials (2.2). A related question is why this quartet eigenvalue symmetry also appears for asymmetric solitons in $\mathcal{P} \mathcal{T}$-symmetric potentials (2.2). In both cases, the linear-stability operator $L$ seems to only admit the symmetry (2.8). Whether this operator also admits another hidden symmetry which assures the eigenvalue symmetry of $\left(\lambda,-\lambda^{*}\right)$ is an open question. If such a hidden $L$ symmetry cannot be found, then how to explain the quartet eigenvalue symmetry of $L$ remains to be seen.

Another important open question concerns the nonlinear stability of solitons in non- $\mathcal{P} \mathcal{T}$-symmetric complex potentials (2.2). In this paper, our focus was the linear (spectral) stability of these solitons, and we showed that these solitons can be linearly stable in a wide range of parameter values. However, it is well known that solitons can be nonlinearly unstable even if they are linearly stable. For instance, in Hamiltonian systems, linearly-stable solitons are nonlinearly unstable if their linear-stability spectrum contains imaginary eigenvalues with negative Krein signatures [43, 44, 46] (these modes with negative Krein signatures are often called negative-energy modes in the physics literature [47]). In our non-Hamiltonian system (2.1), whether these linearly-stable solitons are nonlinearly stable or not is still an open question. One might think that robust evolution simulations of such solitons under random-noise perturbations in Figs. 2(d) and 3(e) should indicate that those solitons are also nonlinearly stable. Such a conclusion is too hasty, since nonlinear instability is often slower and may take longer time to develop [43, 46]. Regarding the issue of nonlinear stability, we should add that this question is also open for solitons in $\mathcal{P} \mathcal{T}$-symmetric systems. A little progress has been made in this direction. In a linear Schrödinger equation with a $\mathcal{P T}$-symmetric potential, which arises when considering the stability of the zero state in Eq. (2.1), a $\mathcal{P} \mathcal{T}$-Krein signature theory was developed recently [48]. This theory can be readily extended to the linear Schrödinger equation with a non- $\mathcal{P} \mathcal{T}$ symmetric potential of the form $(2.2)[32,48]$. Whether a simi- 
lar theory can be developed for solitons in the non-Hamiltonian system (2.1) remains to be seen.

Acknowledgments: This work was supported in part by the Air Force Office of Scientific Research (Grant USAF 955012-1-0244) and the National Science Foundation (Grant DMS1311730).

\section{References:}

[1] S.V. Suchkov, A.A. Sukhorukov, J. Huang, S.V. Dmitriev, C. Lee and Y.S. Kivshar, "Nonlinear switching and solitons in $\mathcal{P} \mathcal{T}$-symmetric photonic systems", arXiv:1509.03378 (2015).

[2] V.V. Konotop, J. Yang and D.A. Zezyulin, "Nonlinear waves in $\mathcal{P T}$ symmetric systems", arXiv:1603.06826 [nlin.PS], 2016.

[3] C.M. Bender and S. Boettcher, "Real spectra in non-Hermitian Hamiltonians having $\mathcal{P} \mathcal{T}$ symmetry", Phys. Rev. Lett. 80, 5243 (1998).

[4] Z.H. Musslimani, K.G. Makris, R. El-Ganainy and D.N. Christodoulides, "Optical solitons in $\mathcal{P} \mathcal{T}$-periodic potentials", Phys. Rev. Lett. 100, 030402 (2008).

[5] C.E. Rüter, K.G. Makris, R. El-Ganainy, D.N. Christodoulides, M. Segev, and D. Kip, "Observation of parity-time symmetry in optics", Nature Phys. 6, 192-195 (2010).

[6] A. Regensburger, C. Bersch, M.A. Miri, G. Onishchukov, D.N. Christodoulides and U. Peschel, "Parity-time synthetic photonic lattices", Nature 488, 167-171 (2012)

[7] B. Peng, S. Özdemir, F. Lei, F. Monifi, M. Gianfreda, G. Long, S. Fan, F. Nori, C. M. Bender, and L. Yang, "Parity-time-symmetric whisperinggallery microcavities", Nat. Phys. 10, 394 (2014).

[8] S. Longhi, "PT-symmetric laser absorber", Phys. Rev. A 82, 031801 (2010).

[9] R. Driben and B.A. Malomed, "Stability of solitons in parity-timesymmetric couplers", Opt. Lett. 36, 4323 (2011).

[10] J. Schindler, A. Li, M. C. Zheng, F. M. Ellis, and T. Kottos, "Experimental study of active LRC circuits with $\mathcal{P} \mathcal{T}$ symmetries," Phys. Rev. A 84, 040101(R) (2011).

[11] S. Klaiman, U. Günther and N. Moiseyev, "Visualization of branch points in $\mathcal{P} \mathcal{T}$-symmetric waveguides," Phys. Rev. Lett. 101, 080402 (2008).

[12] H. Cartarius and G. Wunner, "Model of a $\mathcal{P T}$-symmetric Bose-Einstein condensate in a $\delta$-function double-well potential", Phys. Rev. A 86, 013612 (2012).

[13] C. M. Bender, B. Berntson, D. Parker, and E. Samuel, "Observation of $\mathcal{P} \mathcal{T}$ phase transition in a simple mechanical system", Am. J. Phys. 81, 173179 (2013).

[14] S. Nixon, L. Ge and J. Yang, "Stability analysis for solitons in $\mathcal{P} \mathcal{T}$ symmetric optical lattices," Phys. Rev. A 85, 023822 (2012).

[15] Z. Ahmed, "Real and complex discrete eigenvalues in an exactly solvable one-dimensional complex $\mathcal{P} \mathcal{T}$-invariant potential", Phys. Lett. A 282, 343 (2001).

[16] L. Feng, Y.L. Xu, W.S. Fegadolli, M.H. Lu, J.E.B. Oliveira, V.R. Almeida, Y.F. Chen, and A. Scherer, "Experimental demonstration of a unidirectional reflectionless parity-time metamaterial at optical frequencies", Nature Materials 12, 108 (2013).

[17] L. Feng, Z.J. Wong, R. Ma, Y. Wang, and X. Zhang, "Single-mode laser by parity-time symmetry breaking", Science 346, 972-975 (2014).

[18] H. Hodaei, M.-A. Miri, M. Heinrich, D. N. Christodoulides, M. Khajavikhan, "Parity-timesymmetric microring lasers", Science 346, 975-978 (2014).

[19] H. Wang and J. Wang, "Defect solitons in parity-time periodic potentials", Opt. Express 19, 4030 (2011).

[20] F. K. Abdullaev, Y. V. Kartashov, V. V. Konotop, and D. A. Zezyulin, "Solitons in $\mathcal{P} \mathcal{T}$-symmetric nonlinear lattices", Phys. Rev. A 83, 041805 (2011).

[21] D. A. Zezyulin and V.V. Konotop, "Nonlinear modes in the harmonic PT -symmetric potential”, Phys. Rev. A 85, 043840 (2012).

[22] M. Miri, A.B. Aceves, T. Kottos, V. Kovanis and D.N. Christodoulides,
"Bragg solitons in nonlinear $\mathcal{P} \mathcal{T}$-symmetric periodic potentials", Phys. Rev. A 86, 033801 (2012).

[23] P. G. Kevrekidis, D. E. Pelinovsky, and D. Y. Tyugin, "Nonlinear stationary states in $\mathcal{P} \mathcal{T}$-symmetric lattices", SIAM J. Appl. Dyn. Syst. 12, 1210 (2013).

[24] Y. V. Kartashov, B. A. Malomed, and L. Torner, "Unbreakable $\mathcal{P T}$ symmetry of solitons supported by inhomogeneous defocusing nonlinearity", Opt. Lett. 39, 5641 (2014).

[25] M. Wimmer, A. Regensburger, M.A. Miri, C. Bersch, D.N. Christodoulides and U. Peschel, "Observation of optical solitons in $\mathcal{P T}$ symmetric lattices”, Nature Communications 6, 7782 (2015).

[26] I.V. Barashenkov, D.A. Zezyulin and V.V. Konotop, "Exactly solvable Wadati potentials in the $\mathcal{P} \mathcal{T}$-symmetric Gross-Pitaevskii equation", in NonHermitial Hamiltonians in Quantum Physics (edited by F. Bagarello, R. Passante and C. Trapani, Springer International Publishing, Switzerland, 2016, pp 143-155).

[27] F. Cannata, G. Junker, and J. Trost, "Schrödinger operators with complex potential but real spectrum", Phys. Lett. A 246, 219 (1998).

[28] A.A. Andrianov, M.V. Ioffe, F. Cannata, and J.P. Dedonder, "SUSY quantum mechanics with complex superpotentials and real energy spectra", Int. J. Mod. Phys. A 14, 2675 (1999).

[29] M.A. Miri, M. Heinrich, and D. N. Christodoulides, "Supersymmetrygenerated complex optical potentials with real spectra”, Phys. Rev. A 87, 043819 (2013).

[30] M. Wadati "Construction of parity-time symmetric potential through the soliton theory", J. Phys. Soc. Jpn. 77, 074005 (2008).

[31] E.N. Tsoy, I.M. Allayarov and F. Kh. Abdullaev, "Stable localized modes in asymmetric waveguides with gain and loss", Opt. Lett. 39, 4215 (2014).

[32] S. D. Nixon and J. Yang, "All-real spectra in optical systems with arbitrary gain and loss distributions", Phys. Rev. A 93, 031802(R) (2016).

[33] J. Yang, "Necessity of PT symmetry for soliton families in onedimensional complex potentials", Phys. Lett. A 378, 367-373 (2014).

[34] V. V. Konotop and D. A. Zezyulin, "Families of stationary modes in complex potentials", Opt. Lett. 39, 5535 (2014).

[35] S. D. Nixon and J. Yang, "Bifurcation of soliton families from linear modes in non- $\mathcal{P} \mathcal{T}$-symmetric complex potentials", Stud. Appl. Math. 136, 459-483 (2016).

[36] J. Yang, Nonlinear Waves in Integrable and Nonintegrable Systems (SIAM, Philadelphia, 2010).

[37] Y.S. Kivshar and G.P. Agrawal, Optical Solitons: From Fibers to Photonic Crystals (Academic Press, San Diego, 2003).

[38] L.P. Pitaevskii and S. Stringari, Bose-Einstein Condensation (Oxford University Press, Oxford, 2003).

[39] N. Akhmediev and A. Ankiewicz (Eds), Dissipative Solitons (Springer, Berlin, 2005).

[40] J. Yang, "Can parity-time-symmetric potentials support families of nonparity-time-symmetric solitons?”, Stud. Appl. Math. 132, 332-353 (2014).

[41] J. Yang, "Symmetry breaking of solitons in two-dimensional complex potentials", Phys. Rev. E 91, 023201 (2015).

[42] R.H. Goodman, "Hamiltonian Hopf bifurcations and dynamics of NLS/GP standing-wave modes", J. Phys. A, 44, 425101 (2011).

[43] J. Yang, "A normal form for Hamiltonian-Hopf bifurcations in nonlinear Schrödinger equations with general external potentials", SIAM J. Appl. Math. 76, 598-617 (2016).

[44] T. Kapitula and K. Promislow, Spectral and Dynamical Stability of Nonlinear Waves (Springer, New York, 2013).

[45] J. Yang, "Symmetry breaking of solitons in one-dimensional parity-timesymmetric optical potentials", Opt. Lett. 39, 5547-5550 (2014).

[46] P.G. Kevrekidis, D.E. Pelinovsky, and A. Saxena, "When linear stability does not exclude nonlinear instability”, Phys. Rev. Lett. 114, 214101 (2015).

[47] P. J. Morrison, "Hamiltonian description of the ideal fluid", Rev. Mod. Phys. 70, 467-521 (1998).

[48] S. D. Nixon and J. Yang, "Nonlinear wave dynamics near phase transition in $\mathcal{P} \mathcal{T}$-symmetric localized potentials", arXiv:1506.04445 (2015). 